\title{
Psychiatric co-morbidities in patients of dhat syndrome: A cross-sectional study in a tertiary care centre
}

\author{
Santosh Kumar ${ }^{1, *}$, Harsh Rathi ${ }^{2}$, C. S. Sharma ${ }^{3}$, Vidhata Dixit ${ }^{4}$, Tejinder Singh ${ }^{5}$ \\ ${ }^{1}$ Associate Professor, ${ }^{2,5}$ Junior Resident, ${ }^{3}$ Professor \& HOD, ${ }^{4}$ Lecturer, Dept. of Psychiatry, Rohilkhand Medical College and \\ Hospital, Bareilly, Uttar Pradesh, India
}

*Corresponding Author:

Email: dr.santosh.kr@gmail.com

\begin{abstract}
Dhat syndrome is a culture-bound syndrome characterised by inappropriate and excessive distress of losing semen from one's body and thereby leading to multiple vague psychological and somatic symptoms. It is presumed that several of these symptoms could possibly be of other co-morbid psychiatric disorders. The aim of this study was to assess these co-morbidities with the help of a comprehensive diagnostic tool. Utilizing the purposive sampling technique, a total of 100 patients of Dhat syndrome who fulfilled the inclusion and exclusion criteria of this study were enrolled. They were administered Mini-International Neuropsychiatric Interview (MINI) to assess co-morbid psychiatric disorders. Overall, 37\% of the patients had co-morbidity of at least one psychiatric disorder. Out of these 37 patients with psychiatric co-morbidity, major depression was the most common diagnosis $(43.2 \%)$ followed by generalized anxiety disorder (16.2\%), obsessive compulsive disorder (10.8\%), panic disorder (5.4\%), alcohol dependence (8.1\%), alcohol abuse (5.4\%), other substance abuse (5.4\%), other substance dependence( $2.7 \%$ ), and social anxiety disorder $(2.7 \%)$. We concluded that a co-morbidity of various psychiatric disorders is common in patients of Dhat syndrome and it is not only important to consider Dhat syndrome as a distinct psychiatric entity but also to address various psychiatric conditions co-morbid with it for the sake of overall better outcome.
\end{abstract}

Keywords: Dhat syndrome, Co-morbidity, Psychiatric disorders.

\section{Introduction}

Dhat syndrome is a culture-bound syndrome which has been reported from many countries around the world, with majority of the literature emanating from India. ${ }^{1}$ In India, Wig ${ }^{2}$ coined the term "Dhat syndrome" characterized by vague somatic symptoms of fatigue, weakness, anxiety, loss of appetite and guilt attributed to semen loss through nocturnal emissions, urine and masturbation though there is no evidence of loss of semen. It is commonly seen in young males, of all educational level and socioeconomic status. ${ }^{3}$ Existing literature suggests that the consideration of semen to be important for healthy functioning of male is not only there in Indian subcontinent but also in many Western countries. ${ }^{1}$ It is logical to expect that the cultural belief systems across various countries influence the inappropriate and excessive distress of losing semen from one's body, the core belief system of Dhat syndrome.

Earlier researchers have given varied symptomatic details of Dhat syndrome including vague somatic symptoms, weakness, guilt, lethargy, anxiety, loss of appetite and sleep, multiple body pains, sexual dysfunction, and many other symptoms. ${ }^{4,5}$ These details are still not well described there in the current classificatory system of psychiatric disorders- ICD-10 DCR. ${ }^{6}$ It can be presumed that several of these symptoms could possibly be of other co-morbid physical conditions like sexually transmitted diseases or psychological disorders like depression or anxiety disorders. A recent multi-centric study from India reveals sexual dysfunction to be the most common co- morbidity (present in more than $50 \%$ of the patients) with Dhat syndrome followed by depression and neurotic or somatoform disorders. ${ }^{7}$ One third of patients in this study did not report any other symptoms of psychiatric illness other than Dhat syndrome. ${ }^{7}$ A recent review of world literature suggests depressive and anxiety disorders being the most common co-morbid psychiatric disorders with Dhat syndrome. ${ }^{5}$

A detailed description of psychiatric co-morbidity is always desirable in view of the consideration of Dhat syndrome as an independent psychiatric diagnostic entity. This should happen beyond mere clinical judgement of presence or absence of some common psychiatric co-morbidity expected with the condition as has been a usual clinical practice in current scenario. The present study aims to assess the presence of other co-morchid psychiatric diagnoses in patients of Dhat syndrome with the help of a comprehensive diagnostic tool.

\section{Materials and Methods}

This was a hospital-based cross-sectional observational study conducted at the Department of Psychiatry in a Tertiary Care Centre of Western Uttar Pradesh in India. Permission from Institutional Ethics Committee was obtained prior to study. With the help of purposive sampling technique, the adult ( $>18$ years) male patients with Dhat syndrome who fulfilled the inclusion and exclusion criteria of this study were enrolled after obtaining informed consent. Such patients came on their own (with or without family members) or were referred from other departments for further 
psychiatric evaluation. Diagnosis of Dhat syndrome was made using ICD-10 DCR ${ }^{6}$ diagnostic criteria. The duration of this study was 1 year (November 2015 to October 2016). The patients who were not willing to give written informed consent were excluded from the study. The patients enrolled for the study were assessed on following tools:

Socio-demographic and Clinical Data Sheet (Self Prepared): This was especially prepared for this study to obtain various socio-demographic details like age, religion, marital status, educational level, occupation, residence, socioeconomic status, family type etc. as well as various clinical details like duration of illness, source of referral, source of information about passage of Dhat, frequency of loss of Dhat from body, course of loss of Dhat, knowledge base of patient about Dhat, attribution of loss of Dhat, impact on sexual functioning, reason of present psychiatric consultation etc.

Mini International Neuropsychiatric Interview (MINI) $^{8}$ : This is a short structured diagnostic interview, developed jointly by researchers in the United States and Europe, for DSM-IV and ICD-10 psychiatric disorders. It has 16 diagnoses. It is divided into modules, identified by letters, each corresponding to a diagnostic category. It is a reliable and valid tool.It has been developed to assess the diagnoses of psychiatric patients in less time than other diagnostic interviews such as the Structured Clinical Interview for DSM-IV disorders (SCID), the Composite International Diagnostic Interview (CIDI) or the Schedules for Clinical Assessment in Neuropsychiatry (SCAN) tend to take. MINI has been translated and adopted in many languages around the world and is now a preferred psychiatric structured diagnostic interview instrument employed by mental health professionals and health organizations in more than 100 countries.

Subsequently, the data thus collected were tabulated and statistically analysed using IBM Statistical Package for the Social Sciences version 20 (i.e. SPSS 20) with parametric and nonparametric tests being used as applicable. The level $\mathrm{P}<0.05$ was considered as the cut-off value of significance.

\section{Results}

The mean age of the sample was 27.79 years (standard deviation (SD) \pm 8.05; range 18-60 years).Majority of the subjects were unmarried $(53 \%)$, Hindu (68\%), educated from 1 st to $10^{\text {th }}$ standard (47\%), unskilled workers $(48 \%)$ and from middle social economic status $(51 \%)$, joint family type $(57 \%)$, and urban $(41 \%)$ background of Uttar Pradesh $(63 \%)$ state of India (Table 1).
Table 1: Socio-demographic Details of Patients of Dhat Syndrome (N=100)

\begin{tabular}{|c|c|c|}
\hline \multirow{2}{*}{\multicolumn{2}{|c|}{$\begin{array}{c}\begin{array}{c}\text { Socio-demographic } \\
\text { Variables }\end{array} \\
\end{array}$}} & $\begin{array}{c}\text { Patients of Dhat } \\
\text { Syndrome } \\
(\mathrm{N}=100)\end{array}$ \\
\hline & & Mean \pm SD \\
\hline \multirow{2}{*}{\multicolumn{2}{|c|}{ Age in years (Range: 18-60) }} & $27.79( \pm 8.05)$ \\
\hline & & $\mathrm{n}(\%)$ \\
\hline \multirow[t]{4}{*}{ Religion } & Hindu & $68(68 \%)$ \\
\hline & Muslim & $22(22 \%)$ \\
\hline & Sikh & $6(6 \%)$ \\
\hline & Christian & $4(4 \%)$ \\
\hline \multirow{4}{*}{$\begin{array}{l}\text { Marital } \\
\text { Status }\end{array}$} & Single & $53(53 \%)$ \\
\hline & Married & $44(44 \%)$ \\
\hline & Separated & $2(2 \%)$ \\
\hline & Divorced & $1(1 \%)$ \\
\hline \multirow[t]{5}{*}{ Education } & Illiterate & $9(9 \%)$ \\
\hline & $1^{\text {st }}-10^{\text {th }}$ Std. & $47(47 \%)$ \\
\hline & Pre-University & $19(19 \%)$ \\
\hline & Graduate & $22(22 \%)$ \\
\hline & $\begin{array}{l}\text { Postgraduate or } \\
\text { above }\end{array}$ & $3(3 \%)$ \\
\hline \multirow[t]{3}{*}{ Occupation } & Unemployed & $33(33 \%)$ \\
\hline & $\begin{array}{l}\text { Unskilled } \\
\text { Employment }\end{array}$ & $48(48 \%)$ \\
\hline & $\begin{array}{l}\text { Skilled } \\
\text { Employment }\end{array}$ & $19(19 \%)$ \\
\hline \multirow[t]{3}{*}{ Residence } & Rural & $36(36 \%)$ \\
\hline & Semi Urban & $23(23 \%)$ \\
\hline & Urban & $41(41 \%)$ \\
\hline \multirow[t]{2}{*}{ State } & Uttar Pradesh & $63(63 \%)$ \\
\hline & Uttarakhand & $37(37 \%)$ \\
\hline \multirow{3}{*}{$\begin{array}{l}\text { Socio- } \\
\text { economic } \\
\text { Status }\end{array}$} & Low & $16(16 \%)$ \\
\hline & Middle & $51(51 \%)$ \\
\hline & High & $33(33 \%)$ \\
\hline \multirow{2}{*}{$\begin{array}{l}\text { Family } \\
\text { type }\end{array}$} & Nuclear & $43(43 \%)$ \\
\hline & Joint & $57(57 \%)$ \\
\hline
\end{tabular}

SD: Standard Deviation

Table 2A and Table 2B show clinical details of patients with Dhat syndrome. In majority of the patients, duration of the illness was 7 to 12 months. In majority of the patients, course of the illness was either fluctuating (39\% of the patients) or deteriorating (28\% of the patients) while in $14 \%$ of them it was static and in the rest $19 \%$ of them the course was even improving one. Most of the patients (73\%) presented in the psychiatric outpatient door on their own while only $17 \%$ of them were referred from other specialists of the hospital. Some patients (10\%) visited the psychiatric clinic on suggestion of faith healers in their locality. The majority of the patients came to know about Dhat as a problem from multiple sources like various advertisements (41\%), friends (83\%), local faith healers (75\%), other medical practitioners $(69 \%)$, and family members $(23 \%)$. Some of the patients complained of 
loss of Dhat from body almost daily (39\%) while some of them complained of the problem for a few days in a week $(47 \%)$. Such complaint of loss of Dhat from body was less frequent (as many days in a month) in $14 \%$ of the patients. Majority of the patients attributed loss of Dhat to multiple factors like dirty dreams $(64 \%)$, masturbation (53\%), overindulgence in sex (39\%), and exposure of pornographic materials $(9 \%)$.

Table 2A: Clinical details of Patients of Dhat Syndrome (N=100)

\begin{tabular}{|c|c|c|}
\hline \multicolumn{2}{|c|}{ Clinical Variables } & $\begin{array}{c}\text { Patients of } \\
\text { Dhat }\end{array}$ \\
\hline \multirow{4}{*}{$\begin{array}{l}\text { Duration of } \\
\text { Dhat } \\
\text { syndrome }\end{array}$} & 0-6 months & $11(11 \%)$ \\
\hline & 7-12 months & $53(53 \%)$ \\
\hline & $2-4$ years & $29(29 \%)$ \\
\hline & $>4$ years & $7(7 \%)$ \\
\hline \multirow{4}{*}{$\begin{array}{l}\text { Course of } \\
\text { Dhat } \\
\text { syndrome }\end{array}$} & Fluctuating & $39(39 \%)$ \\
\hline & Deteriorating & $28(28 \%)$ \\
\hline & Improving & $19(19 \%)$ \\
\hline & Static & $14(14 \%)$ \\
\hline \multirow{3}{*}{$\begin{array}{l}\text { Source of } \\
\text { referral for } \\
\text { psychiatric } \\
\text { consultation }\end{array}$} & Self & $73(73 \%)$ \\
\hline & $\begin{array}{l}\text { Other medical / } \\
\text { surgical specialists }\end{array}$ & $17(17 \%)$ \\
\hline & Faith healers & $10(10 \%)$ \\
\hline \multirow{5}{*}{$\begin{array}{l}\text { Source of } \\
\text { Information } \\
\text { about Dhat } \\
\text { as a problem }\end{array}$} & $\begin{array}{l}\text { Various } \\
\text { advertisements }\end{array}$ & $41(41 \%)$ \\
\hline & Friends & $83(83 \%)$ \\
\hline & Family members & $23(23 \%)$ \\
\hline & $\begin{array}{l}\text { Medical } \\
\text { practitioner }\end{array}$ & $69(69 \%)$ \\
\hline & Faith healers & $75(75 \%)$ \\
\hline \multirow{3}{*}{$\begin{array}{l}\text { Frequency of } \\
\text { loss of Dhat } \\
\text { from body }\end{array}$} & Almost daily & $39(39 \%)$ \\
\hline & $\begin{array}{l}\text { Few days in a } \\
\text { week }\end{array}$ & $47(47 \%)$ \\
\hline & $\begin{array}{l}\text { Many days in a } \\
\text { month }\end{array}$ & $14(14 \%)$ \\
\hline \multirow{5}{*}{$\begin{array}{l}\text { Patient } \\
\text { attributes } \\
\text { loss of Dhat } \\
\text { to }\end{array}$} & Masturbation & $53(53 \%)$ \\
\hline & Dirty dreams & $64(64 \%)$ \\
\hline & $\begin{array}{l}\text { Overindulgence in } \\
\text { sex }\end{array}$ & $39(39 \%)$ \\
\hline & $\begin{array}{l}\text { Spontaneous } \\
\text { passage of Dhat } \\
\text { during micturition } \\
\text { as evidenced by } \\
\text { turbid urine }\end{array}$ & $13(13 \%)$ \\
\hline & $\begin{array}{l}\text { Exposure to } \\
\text { pornographic } \\
\text { materials }\end{array}$ & $9(9 \%)$ \\
\hline
\end{tabular}

MINI: Mini-International Neuropsychiatric Interview

The patients had multiple points of view of importance of Dhat in their body. Overall $93 \%$ of the patients claimed loss of Dhat to be responsible for loss of energy while $89 \%$ of all the patients recognized the problem to be harmful to body. Loss of Dhat can reduce longevity in men was reported by $79 \%$ of all the patients while $63 \%$ of the patients considered such loss to be responsible for sexual dysfunctions. Overall $62 \%$ of the patients had a view that loss of Dhat to have bad impact on external genitalia too while 58\% of all patients considered such loss to have bad impact on fertility or potency as well. A co-morbidity of Koroanother culture bound syndrome was found in 19\% of the patients. Overall $37 \%$ of all patients of Dhat syndrome had co-morbidity of at least one of psychiatric disorders.

Table 2B: Clinical details of Patients ofDhat Syndrome (N=100)

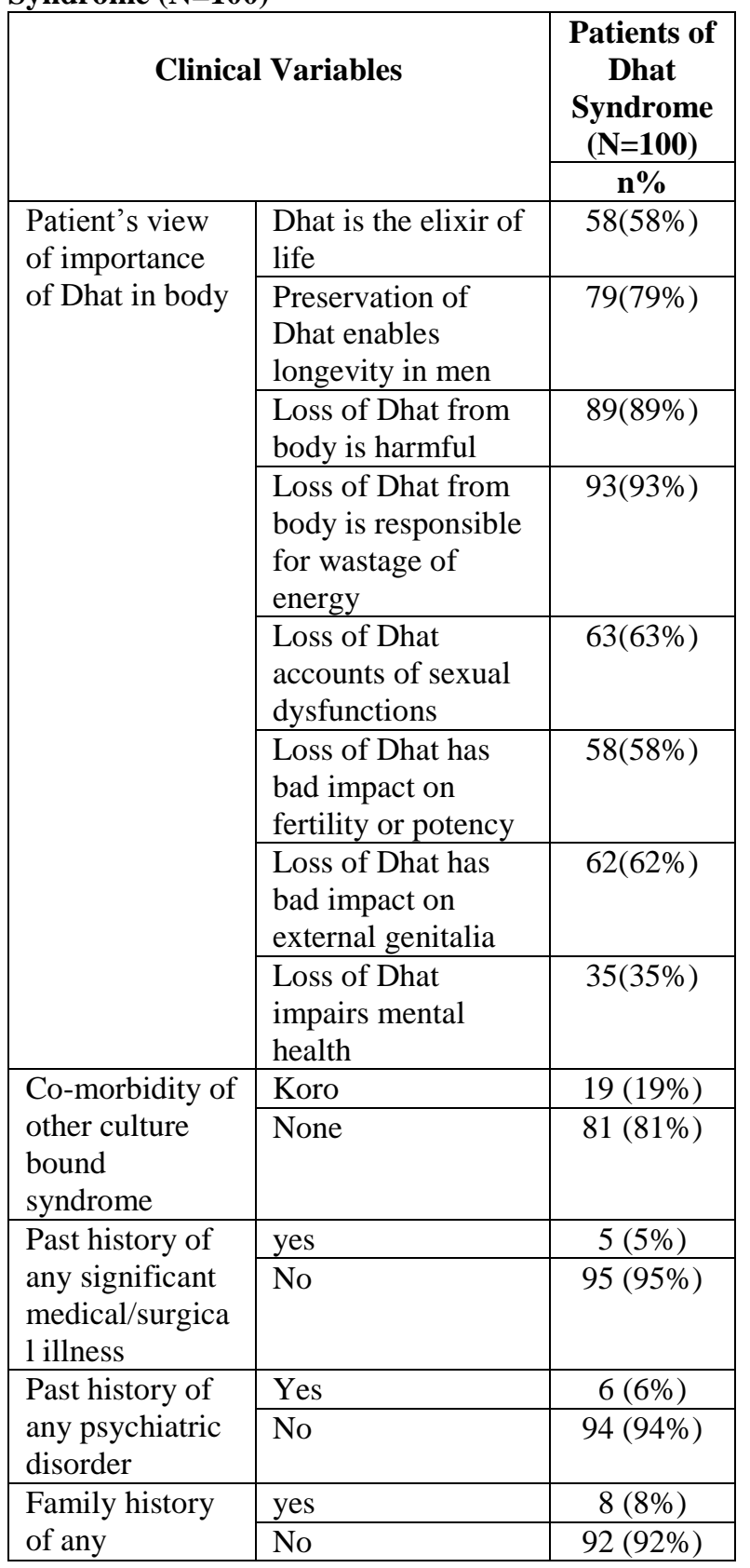




\begin{tabular}{|l|l|l|}
\hline $\begin{array}{l}\text { psychiatric } \\
\text { disorder }\end{array}$ & & \\
\hline $\begin{array}{l}\text { Presence of any } \\
\text { psychiatric } \\
\text { comorbidity on } \\
\text { MINI }\end{array}$ & Yes & $37(37 \%)$ \\
\cline { 2 - 3 } & & $63(63 \%)$ \\
\hline
\end{tabular}

MINI: Mini International Neuropsychiatric Interview

Fig. 1 shows the distribution of co-morbid psychiatric disorders, as assessed on MINI (Mini International Neuropsychiatric Interview) in patients of Dhat syndrome. Overall, Major depression (43.2\%) was the most common psychiatric co-morbidity. Other less frequent co-morbid psychiatric conditions were as follows: Generalized anxiety disorder- $16.2 \%$, Obsessive compulsive disorder- $10.8 \%$, Panic disorder5.4\%, Alcohol dependence- $8.1 \%$, Alcohol abuse$5.4 \%$, other substance abuse- $5.4 \%$, other substance dependence- $2.7 \%$, and Social anxiety disorder- $2.7 \%$.

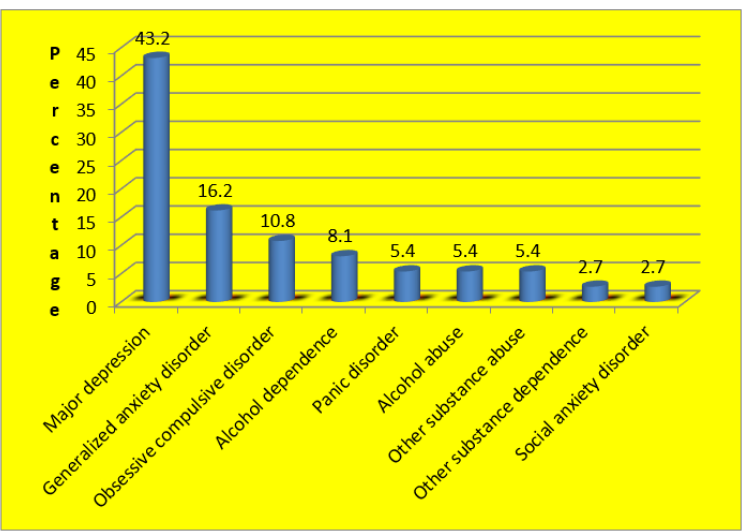

Fig. 1: Psychiatric Co-morbidities in Patients of Dhat Syndrome

Patients of Dhat syndrome were also compared on the basis of presence or absence of any psychiatric comorbidity. The patients with psychiatric co-morbidity $(\mathrm{N}=37)$ were comparable to those without psychiatric co-morbidity $(\mathrm{n}=63)$ in respect to all sociodemographic details except family type where the former belonging to nuclear family type were significantly higher in number than the later belonging to nuclear family type $(67.6 \%$ vs $41.3 \%, \chi 2=6.451$, $\mathrm{p}<.05)$. Further the patients with psychiatric comorbidity $(\mathrm{N}=37)$ were comparable to those without psychiatric co-morbidity $(n=63)$ in respect to all clinical details except the source of referral where the former presented themselves to psychiatric OPD in significantly higher number than the later presented themselves to psychiatric OPD $(86.5 \%$ vs $65.1 \%$, $\chi 2=8.608, \mathrm{p}<.05)$.

\section{Discussion}

This was a hospital based cross-sectional observational study to assess psychiatric co-morbidity in patients of Dhat syndrome with the help of a comprehensive diagnostic tool- MINI i.e. Mini International Neuropsychiatric Interview. ${ }^{8}$ Overall, 37\% of the patients had co-morbidity of at least one psychiatric disorder. Out of these 37 patients with psychiatric co-morbidity, Major depression was the most common diagnosis (43\%) followed by Generalized anxiety disorder (16\%) and Obsessive compulsive disorder (10\%). Other conditions like Panic disorder, Social anxiety disorder, Alcohol use disorder (abuse or dependence) or other substance disorder (abuse or dependence) were less common diagnoses each present in less than $10 \%$ of these patients with psychiatric co-morbidity. The rates of major depression (40-66\% in earlier studies) and anxiety disorders (20$38 \%$ in earlier studies) in the present sample were similar to that reported in previous studies. ${ }^{9,10}$ In keeping with the finding of our study, a very recent study from North India, also utilizing MINI (i.e. Mini International Neuropsychiatric Interview), finds psychiatric co-morbidity to be present in about one third of all patients of Dhat syndrome. ${ }^{11}$ However, that study finds major depression (14\%), anxiety disorders (11\%) and substance use disorders (7\%) to be present in smaller number on patients of Dhat syndrome. This can be due to their different sample selection based on selfproposed diagnostic criteria for Dhat disorder based on the opinions of various stakeholders - patients, psychiatrists, other medical specialists, and traditional healers. ${ }^{11}$

Majority of the patients of Dhat syndrome were young unmarried male of formal education of matriculation to graduation and belonged to middle socioeconomic joint family of urban or rural background in western part of Uttar Pradesh (India). Characteristically $44 \%$ of the patients were married as well. Further, in comparison to the patients of Dhat syndrome without psychiatric co-morbidity, those with psychiatric co-morbidity were more in number to come from nuclear family as well as to consult themselves to psychiatrist. In view of predominant co-morbidity of depression, various anxiety disorders and substance use disorders, it appears reasonable that such patients were more eager to seek any psychiatric help.

In present study the patients reported loss of Dhat during multiple situations like dirty dreams (64\%), masturbation $(53 \%)$, overindulgence in sex $(39 \%)$, and exposure to pornographic materials (9\%). The rate of multiple situations of loss of Dhat appeared similar in a multicentric Indian study ${ }^{7}$ except exposure to pornographic materials. There it was found that around half of all patients attributed loss of Dhat to reading or watching pornography. Such a large difference in accepting a problem in context of pornography in our study may be due to specific guilt ridden connotation of pornography which can be expected in this part of India not matching with any national average. A somewhat unusual finding of passage of Dhat through the anal route in a considerable number of patients has also been 
reported by a recent Indian study ${ }^{11}$ but our study could not find this in any of our sample.

It appears reasonable that our cultural perspective does shape the experience and understanding of mental illness. Keeping in view of these cultural influences, Yap ${ }^{12}$ coined the term 'culture-bound syndrome' as an episodic, dramatic and discrete pattern of behavioural reaction specific to a particular community that articulates both personal predicament and public concerns. Our study tried to find it out all relevant socio-demographic and clinical details of the patients of Dhat syndrome with the help of a self-designed semistructured performa with an aim to explore relevant cultural influences of the region on the clinical profile of the patients. The patients had multiple exaggerated views of importance of Dhat which is beyond current scientific explanation of semen in our body. Majority of the subjects considered loss of semen (i.e. Dhat) from their body via any mean to be responsible for loss of energy, harm to body, reduced longevity, sexual dysfunctions and so on. The literature from Indian subcontinent suggests that these subjects get exposed to such information since early age when they start gaining sexual knowledge from various sources like friends, colleagues or relatives based on on-going cultural belief system. One of the authors from India pointed out that a fear of semen loss and its cure are propagated by local practitioners of medicine (i.e. vaids and hakims) and advertised everywhere on walls, on television, in newspapers and on roadside hoardings in most of the northern Indian cities. ${ }^{4}$

In a country like India, consultation to Psychiatrists is stigmatized to a greater extent and the fear laden persons with loss of Dhat as a problem prefer to visit Sexually Transmitted Diseases (STD) clinics run by urologists and physicians rather than to approach psychiatrists directly. In our study it was found that the patients considered Dhat as a problem on the basis of such information from multiple sources in their surroundings out of which feedbacks from friends, local medical practitioners and local faith-healers were most noticeable. However, referral of the patients for psychiatric consultation from either faith-healers or local medical practitioners was less common. The majority of the patients visited psychiatric outpatient department on their own. This indirectly shows increasing trend of psychiatric consultation at present for problems of such nature.

The current classificatory system for psychiatric diagnoses i.e. ICD-10 DCR ${ }^{6}$ defines Dhat Syndrome under a broader category- 'F48.8 Other specified neurotic disorders', where other culture bound syndromes like Koro and Latah have also been placed. This category looks these syndromes as mixed disorders of behaviour, beliefs, and emotions which are of uncertain aetiology and sonological status and which occur with particular frequency in certain cultures. Because of the strong association of these syndromes with locally accepted cultural beliefs and patterns of behaviour they are probably best regarded as not delusional. However, a full clinical description of Dhat syndrome in terms of specific situations of loss of Dhat, reasons and consequences of loss of Dhat as well as details of associated psychological and somatic morbidity with loss of Dhat has not been given by ICD10. Possibly this is the reason that the nosological validity of this disorder is at times questioned and considered by some ${ }^{15}$ to be a cultural variant of depression.

A recent nationwide multicentric study from India emphasizes Dhat syndrome to be found across multiple cultures of the country as well as to occur without syndromal depression and anxiety disorders. ${ }^{7}$ Some authors argue that Dhat syndrome should not be considered as a 'culture bound syndrome' because of its wider geographic prevalence, rather it should be seen as a culturally determined idiom of distress. ${ }^{1} \mathrm{~A}$ few others see Dhat syndrome as an independent disorder, which has its own clinical manifestations. ${ }^{3}$ Because World Health Organization (WHO) is in the process of revising the ICD-10, we should wait for a proper and comprehensive clinical description of Dhat syndrome.

Overall, the present study gives a fair sociodemographic and clinical details of patients of Dhat syndrome as well as it emphasizes that a co-morbidity of other psychiatric disorders in these patients is a common occurrence. The results of this study are in line with many different research works done both in India and abroad. However, the purposive sampling from a single outpatient clinic of a tertiary care hospital may not be representative of the patients of Dhat syndrome in the general population and thereby it limits the generalization of findings of this study.

\section{Conclusion}

The present study concludes that it is common for patients of Dhat syndrome to have a co-morbidity of other psychiatric disorders to be present in around one third of such patients. Overall, major depression is the most common of these co-morbidities followed by anxiety disorders (like generalized anxiety disorder, obsessive compulsive disorder, panic disorder and social anxiety disorder) and substance use disorders (like alcohol abuse or dependence and other substance abuse or dependence). Thus it is important for healthcare providers not only to consider Dhat syndrome as a distinct psychiatric entity but also to address various psychiatric conditions co-morbid with it during management as well as policy making for overall better outcome of such patients. Future studies should apply the instrument on different samples (larger, different settings, and geographical areas) by different investigators and with more items. 


\section{References}

1. Sumathipala, A, Sribaddana, SH, Bhugra, D. Culture bound syndromes: The story of Dhat syndrome. $\mathrm{Br} \mathrm{J}$ Psychiatry 2004;184:200-9.

2. Wig NN. Problem of mental health in India. J Clin Social Psychiatry 1960;17:48-53.

3. Grover S, Avasthi A, Gupta S, Dan A, Neogi R.

Phenomenology and beliefs of patients with Dhat Syndrome: A nationwide multicentric study. Int J Soc Psychiatry 2016;62(1):57-66.

4. Prakash O. Lessons for postgraduate trainees about Dhat syndrome. Indian J Psychiatry 2007;49:208-10.

5. Deb KS, Balhara YP. Dhat syndrome: A review of the world literature. Indian J Psychol Med 2013;35:326-31.

6. World Health Organization. The ICD-10 Classification of Mental and Behavioural Disorders; Clinical Description and Diagnostic Guidelines. Geneva: WHO;1992.

7. Grover S, Avasthi, A, Gupta S, Dan A, Neogi R. Comorbidity in patients with Dhat syndrome: A nationwide multicentric study. J Sex Med 2015;12:1398-401.

8. Sheehan DV, Lecrubier Y, Sheehan KH, Amorim P, Janavs J, Weiller E, et al. The Mini-International Neuropsychiatric Interview (MINI): The development and validation of a structured diagnostic psychiatric interview for DSM-IV and ICD-10. J Clin Psychiatry1998;59(Suppl 20):22-57.

9. Gautham M, Singh R, Weiss H, Brugha R, Patel V, Desai $\mathrm{NG}$, et al. Socio-cultural, psychosexual and biomedical factors associated with genital symptoms experienced by men in rural India. Trop Med Int Health 2008;13:384-95.

10. Prakash O, Rao TS. Sexuality research in India: An update. Indian J Psychiatry 2010;52Suppl 1:S260-3.

11. Prakash S, Sharan P, Sood M. A study on phenomenology of Dhat syndrome in men in a general medical setting. Indian J Psychiatry 2016;58:129-41.

12. Yap PM. Classification of the culture-bound reactive syndromes. Aust N Z J Psychiatry 1967;1:172-9.

13. Mumford DB. The 'Dhat syndrome': A culturally determined symptom of depression? Acta Psychiatr Scand 1996;94:163-7. 